Article

\title{
CRISPR-Mediated Non-Viral Site-Specific Gene Integration and Expression in T Cells: Protocol and Application for T-Cell Therapy
}

\author{
Zelda Odé ${ }^{1}$, Jose Condori ${ }^{1}$, Nicolas Peterson ${ }^{2}$, Sheng Zhou ${ }^{3}$ and Giedre Krenciute ${ }^{1, * \mathbb{D}}$ \\ 1 Department of Bone Marrow Transplantation and Cellular Therapy, St Jude Children's Research Hospital, \\ Memphis, TN 38105, USA; zelda.ode@gmail.com (Z.O.); jose.condori@stjude.org (J.C.) \\ 2 Graduate School of Biomedical Sciences, St Jude Children's Research Hospital, Memphis, TN 38105, USA; \\ nicolas.peterson@stjude.org \\ 3 Experimental Cellular Therapeutics Lab, St Jude Children's Research Hospital, Memphis, TN 38105, USA; \\ sheng.zhou@stjude.org \\ * Correspondence: giedre.krenciute@stjude.org; Tel.: +1-901-595-2188
}

Received: 30 April 2020; Accepted: 24 June 2020; Published: 26 June 2020

\begin{abstract}
T}$ cells engineered with chimeric antigen receptors (CARs) show great promise in the treatment of some cancers. Modifying T cells to express CARs generally relies on T-cell transduction using viral vectors carrying a transgene, resulting in semi-random DNA integration within the T-cell genome. While this approach has proven successful and is used in generating the Food and Drug Administration (FDA, USA) approved B-lymphocyte antigen CD19-specific CAR T cells, it is possible the transgene could integrate into a locus that would lead to malignant transformation of the engineered T cells. In addition, manufacturing viral vectors is time-consuming and expensive. One way to overcome these challenges is site-specific gene integration, which can be achieved through clustered regularly interspaced short palindromic repeat (CRISPR) mediated editing and non-viral DNA, which serves as a template for homology-directed repair (HDR). This non-viral gene editing approach provides a rapid, highly specific, and inexpensive way to engineer $\mathrm{T}$ cells. Here, we describe an optimized protocol for the site-specific knock-in of a large transgene in primary human $\mathrm{T}$ cells using non-viral double stranded DNA as a repair template. As proof-of-principle, we targeted the T-cell receptor alpha constant (TRAC) locus for insertion of a large transgene containing green fluorescence protein (GFP) and interleukin-15 (IL-15). To optimize the knock-in conditions we tested template DNA concentration, homology arm length, cell number, and knock-in efficiency over time. We then applied these established guidelines to target the TRAC or interleukin-13 (IL-13) locus for the knock-in of synthetic molecules, such as a CAR, bispecific T-cell engager (BiTE), and other transgenes. While integration efficiency depends on the targeted gene locus and selected transgene, this optimized protocol reliably generates the desired insertion at rates upwards of $20 \%$. Thus, it should serve as a good starting point for investigators who are interested in knocking in transgenes into specific loci.
\end{abstract}

Keywords: CAR; T cell; non-viral; HDR; CRISPR-Cas9; knock-in; TRAC

\section{Introduction}

T-cell therapies are widely being explored for the treatment of several clinical indications including multiple types of cancer and autoimmune diseases [1,2]. One therapy involves adoptive transfer of patient-derived $\mathrm{T}$ cells that have been engineered to express chimeric antigen receptors (CARs) specific for tumor-associated antigens. CAR engineered $\mathrm{T}$ cells are then infused back into the patient and are able to recognize and kill tumor cells expressing targeted antigens on their surface. CAR T-cell therapy has produced outstanding results in CD19 antigen-positive hematological B-cell malignancies resulting 
in complete response rates of about $83 \%$ in relapsed/refractory B-cell acute lymphoblastic leukemia (ALL) patients [3-6].

Current preclinical and clinical production of CAR $\mathrm{T}$ cells relies to a great extent on T-cell transduction using viral vectors (e.g., retrovirus, lentivirus) to deliver transgenes of interest. However, a CAR transgene delivered to a $\mathrm{T}$ cell by viral transduction is subjected to random integration into the host DNA. This can lead to unpredictable and variable expression of the transgene. In addition, random integration could lead to a malignant transformation if the transgene is integrated into an oncogenic locus $[7,8]$. Finally, viral vector production is time-consuming ( $>6$ months), expensive, and poses biosafety challenges [9-13].

Genetic engineering approaches that aim to integrate a therapeutic gene into a targeted locus have the potential to solve the problems associated with random integration. Site-specific gene integration can be achieved with the use of gene-editing tools (e.g., CRISPR-Cas9, transcription activator-like effector nucleases (TALEN), zinc finger nucleases (ZFN), meganucleases), which results in DNA double-strand breaks (DSBs) and homology-directed repair (HDR) when a donor DNA template is provided. This approach is known as gene knock-in (KI). In addition, a newly emerging Cas 9 base editor technology, which can make gene modifications without DSBs, holds great promise for gene KI in T cells [14]. More and more KI studies are emerging using CRISPR-Cas9 in human T cells to develop clinically useful engineered $\mathrm{T}$ cells. In one of the first proof-of-concept studies, a short 12 base pairs (bp) oligonucleotide flanked by $90 \mathrm{bp}$ homology arms was used to create a targeted gene modification at the C-X-C chemokine receptor type 4 (CXCR4) locus resulting in 20\% knock-in efficiency and creating a targeted gene modification [15]. In another study, applying site-specific DNA integration, programmed cell death protein 1 (PD-1) expression was reduced by $58.6 \%$ using a single-stranded DNA oligo (99 bp) carrying a single nucleotide deletion mutation [16].

Recently, Hale et al. reported successful, targeted CD19-CAR integration into the T-cell receptor alpha constant (TRAC, 42\% of HDR) and C-C chemokine receptor type 5 (CCR5; 37\% of HDR) loci in $\mathrm{T}$ cells using megaTAL nuclease approach and recombinant adeno-associated virus (AAV) for HDR template delivery [17]. Shortly after, Eyquem and colleagues also reported a successful CD19-CAR knock-in to the TRAC locus using CRISPR-Cas9 gene editing [18]. This resulted in improved and consistent CAR expression in T cells, decreased baseline (tonic) signaling, and increased anti-tumor activity in vivo when compared to the CAR T cells generated by viral transduction [18]. Similar to the other group, they also used an AAV vector to deliver donor DNA to T cells for HDR-mediated site-specific integration. Such an approach is time consuming, expensive, and labor-intensive because it requires cloning template DNA into the appropriate vector and producing a high titer viral supernatant prior to gene editing. To overcome these obstacles, Roth and colleagues characterized a different method of HDR template delivery. Instead of employing a viral vector, they utilized non-viral double-stranded DNA (dsDNA) as an HDR template, which was generated via conventional PCR amplification [19]. This method results in high-efficiency knock-in and is substantially cheaper and faster than using a viral vector-based delivery. Thus, it has the potential to reduce costs and time for generating targeted gene modifications in human $\mathrm{T}$ cells for therapeutic use.

Here, we describe an optimized step-by-step protocol for a CRISPR-Cas9-mediated knock-in strategy using a dsDNA as a donor DNA template to insert a transgene of interest into a specific region in the T-cell genome. For our knock-in experiments we used non-viral DNA as an HDR template as described in Roth et al. [19]. For the protocol optimization steps, we targeted the TRAC locus as the insertion site of our transgene. This genomic region has been used for multiple CRISPR-Cas9-mediated gene integration studies and has been shown to be a stable and safe integration site [17-20]. Overall, we demonstrated an efficient integration of a large transgene construct into the TRAC locus and determined optimal conditions for CRISPR-Cas9-mediated knock-in. We also showed that synthetic gene integration into the $I L-13$ locus of $T$ cells can create an inducible system controlled by T-cell activation. 


\section{Results}

\subsection{Gene Knock-In Using Primary T Cells: Overview}

For protocol establishment, we chose primary human T cells as our target cells because they are clinically relevant. To optimize knock-in conditions we targeted the TRAC locus for gene insertion, which has been previously explored for the knock-in of several genes [18,19]. Integration of a promoterless transgene into the TRAC locus will disrupt TRAC expression. However, the endogenous promoter will continue to drive the expression of the newly inserted synthetic gene. For successful integration of a large transgene, the following elements have to be considered: (1) Target site and guide RNAs (gRNAs), (2) transgene design, (3) donor DNA length, type (single stranded DNA (ssDNA), double-stranded DNA (dsDNA), or plasmid) and delivery, (4) detection and efficiency of the knock-in, and (5) T-cell viability (Figure 1). In our proof-of-concept study, we used two transgenes, IL-15 and mClover3, separated by a $2 \mathrm{~A}$ sequence. When integrated into the T-cell genome, gene-edited T cells will express mClover3 fluorescent protein [21] and can be readily detected by flow cytometry (green fluorescence protein (GFP) channel). Secretion of IL-15 can be analyzed by ELISA. Importantly, the IL-15 and mClover3 expression cassette is close to the size of a CAR molecule. Hence, our findings can be readily applied for CAR knock-in into human T cells. To optimize the knock-in conditions we evaluated template DNA concentration, cell number, homology arm length, and knock-in efficiency over time, all of which are discussed in detail below. With the optimized protocol, we were able to achieve up to $60 \%$ knock-in efficiency and establish guidelines for the gene knock-in in T cells, accelerating the process of T-cell engineering.

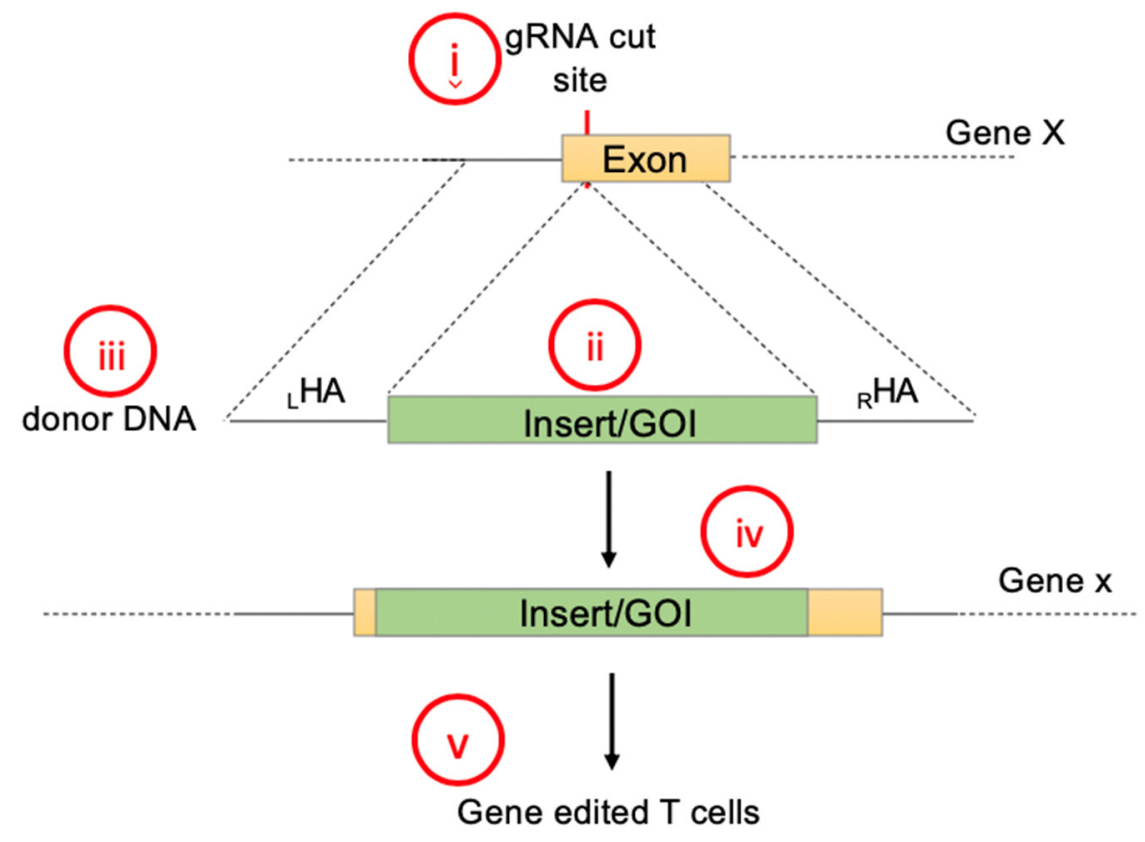

Figure 1. Steps to consider for transgene knock-in using non-viral DNA delivery: (i) Target site and guide RNAs, (ii) transgene design, (iii) donor DNA length, DNA type and delivery method, (iv) detection and efficiency of the knock-in, and (v) viability and performance of genetically engineered $\mathrm{T}$ cell containing the gene of interest.

\subsection{Designing Donor DNA}

While there are many published studies on gene editing using CRISPR-Cas9-mediated knock-in, there are no universal guidelines on how to design a donor/template DNA for HDR-mediated gene insertion. Donor DNA consists of a gene of interest (GOI) flanked by left and right homology arms ( ${ }_{\mathrm{L}} \mathrm{HA}$ and $\left.{ }_{\mathrm{R}} \mathrm{HA}\right)$, which are sequences homologous to the target locus (Figure 2a). In addition, the donor DNA can also include other elements such as a promoter, enhancer, and 2A self-cleaving peptide 
or internal ribosome entry site (IRES) sequence at the $5^{\prime}$ and poly(A) signal at the $3^{\prime}$ end. HAs are designed to flank the Cas9 cutting site, with equal length HAs of up to $800 \mathrm{bp}$ per side (Figure 2a,b). Based on these rules, our final donor DNA for the TRAC locus contained the following parts: $400 \mathrm{bp}$

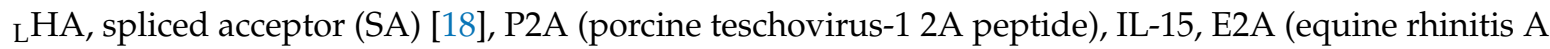
virus $2 \mathrm{~A}$ peptide), mClover3, poly(A) (bovine growth hormone polyadenylation signal), and $400 \mathrm{bp}$ ${ }_{\mathrm{R}} \mathrm{HA}$ (Figure 2c). We included a P2A sequence at the $5^{\prime}$ end to separate our transgene from a possible fusion to the endogenous gene, and a poly(A) sequence at the $3^{\prime}$ end for efficient termination as simple STOP codon might not be sufficient. Lastly, we also mutated the protospacer adjacent motif (PAM) sequence in the ${ }_{\mathrm{L}} \mathrm{HA}$ to inhibit the Cas9 enzyme from repeatedly cutting the DNA in this location. The construct was then synthesized by GeneArt and inserted into the pMA plasmid. This plasmid was then used as a template for PCR reaction to amplify dsDNA and to generate donor DNA for HDR-mediated gene knock-in using CRISPR-Cas9.

a

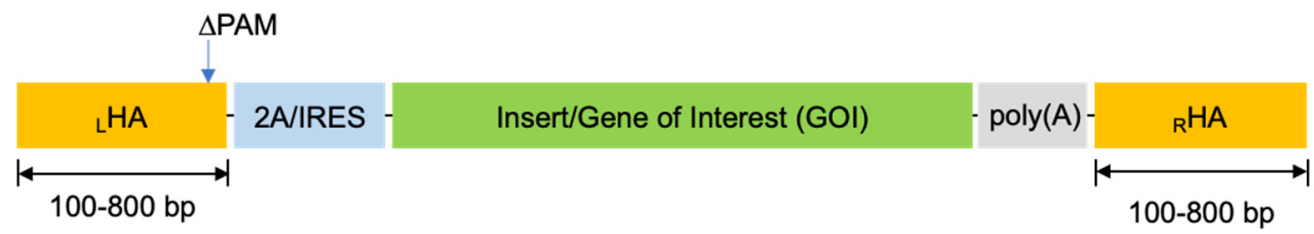

b

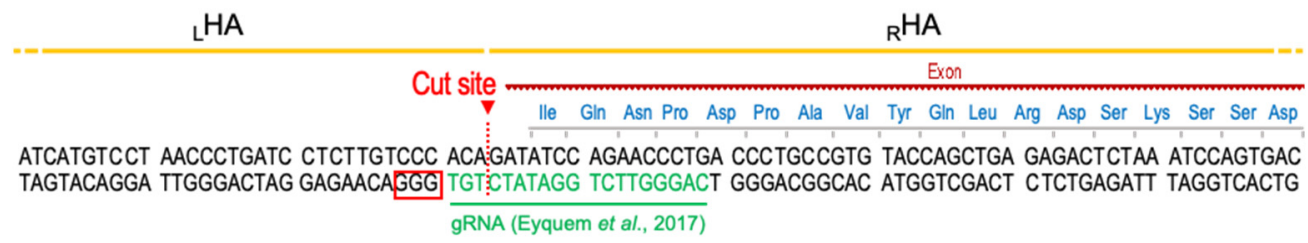

C

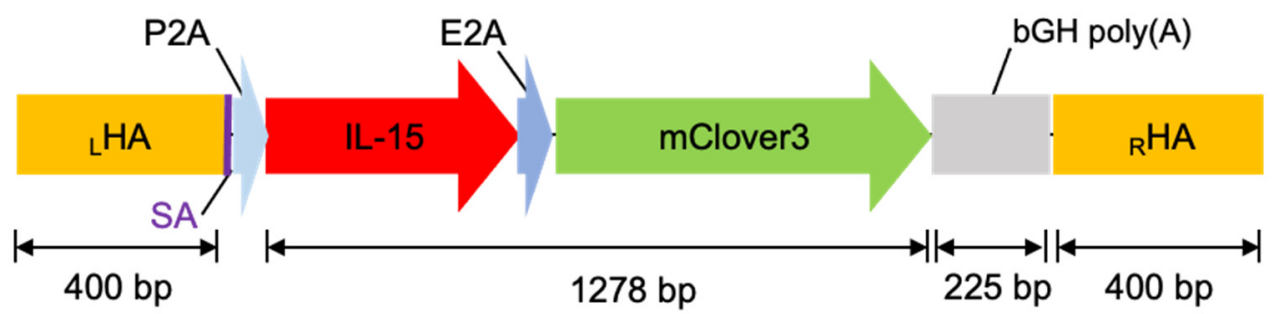

Figure 2. Components of non-viral transgene cassette for expression under endogenous promoter: (a) Schematic of non-viral cassette encoding a gene of interest surrounded by a 2A/IRES at the $5^{\prime}$ end for cleavage GOI from endogenous gene, and poly (A) region at the $3^{\prime}$ end for complete termination; ${ }_{\mathrm{L}} \mathrm{HA}$, left homology arm; ${ }_{\mathrm{R}} \mathrm{HA}$, right homology arm; $\triangle \mathrm{PAM}$, mutated PAM sequence of guide (g) RNA. (b) Design of homology arms (HAs) surrounding the cut site of gRNA targeting human TRAC locus. PAM sequence is indicated in the red box. (c) Scheme of the construct used for the study. It consists of IL-15 and mClover3 for dual transgene expression; SA, splice acceptor; P2A, "self-cleaving" 2A peptide derived from porcine teschovirus-1; E2A, "self-cleaving" 2A peptide derived from equine rhinitis A virus; $\mathrm{bGH}$ poly (A), bovine growth hormone polyadenylation signal.

\subsection{Donor DNA Amplification, Purification, and Concentration}

An overview of the donor DNA amplification, purification, and concentration protocol is shown in Figure 3a and detailed knock-in protocol is provided in the Supplementary Materials. Briefly, primers to amplify donor DNA were designed using SnapGene ${ }^{\mathrm{TM}}$ for different homology arm lengths $(100,200,300$, and $400 \mathrm{bp})$ for insertion in the TRAC gene locus. The dsDNA was generated by PCR amplification using CloneAmp HiFi Taq polymerase (Takara Bio, Mountain View, CA, USA), forward and reverse primers, plasmid DNA, and nuclease-free water in a total of $50 \mu \mathrm{L}$ reaction volume and ran on the ProFlex ${ }^{\mathrm{TM}}$ thermocycler (Thermofisher, Waltham, MA, USA). Two PCR reaction products were combined and separated by electrophoresis on $1 \%$ agarose gel for DNA size confirmation and gel 
purification. To generate high amounts of dsDNA, eight PCR reactions were run in total and two of these reactions were combined in one gel slot.

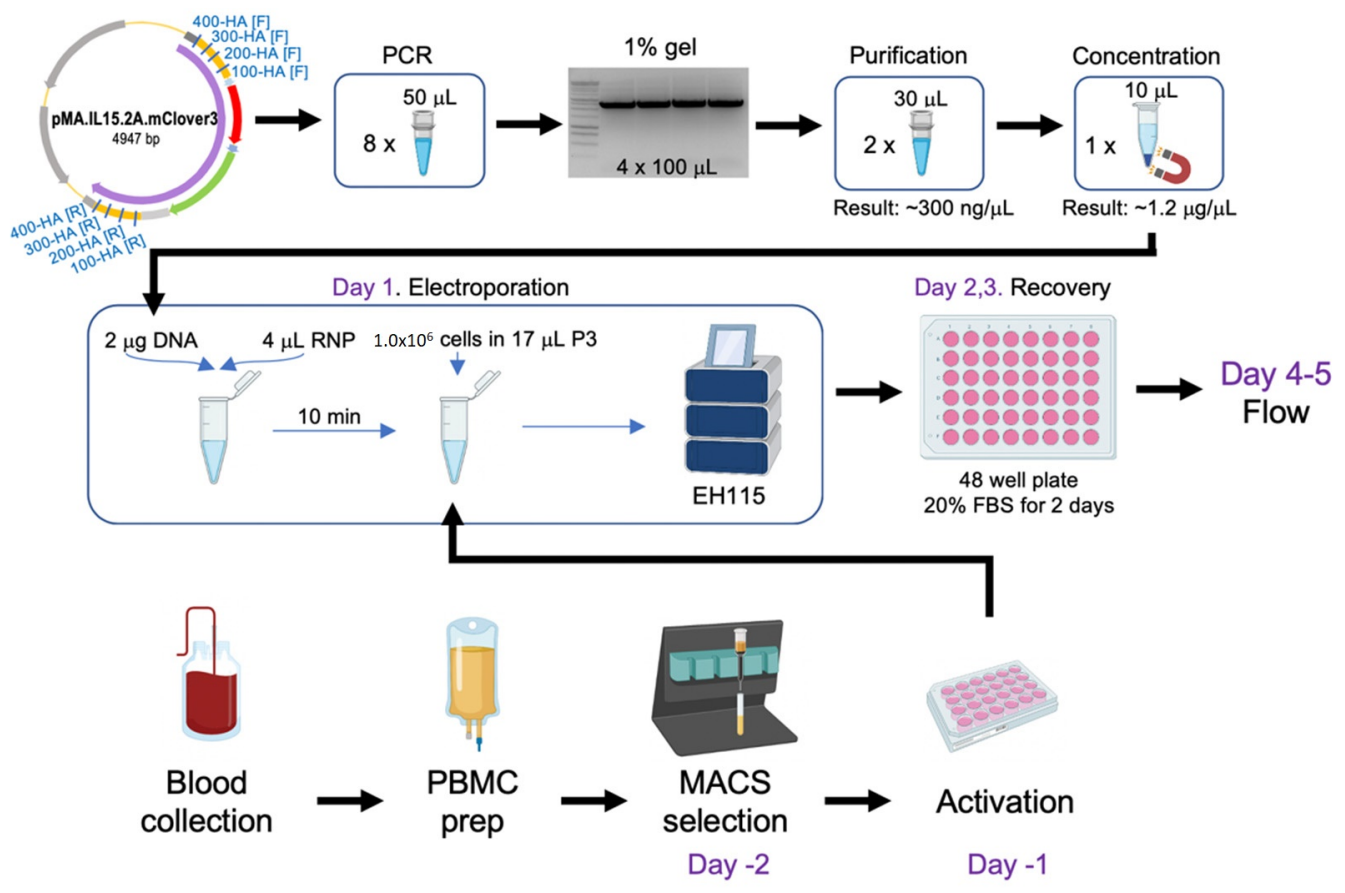

d
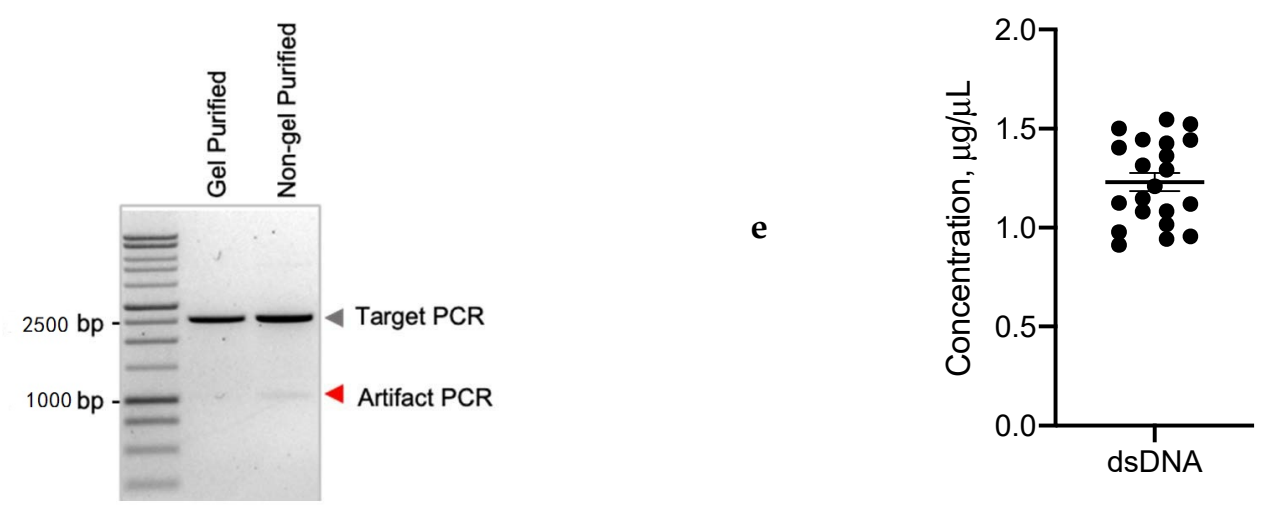

Figure 3. Overview of the steps to generate transgene knock-in in T cell: (a) Molecular steps considering vector design, amplification, purification, and concentration DNA. (b) Electroporation of RNA ribonucleoproteins (RNPs) and donor template to T cells using Lonza instrument. (c) Human T-cell preparation before electroporation. Complete and detailed knock-in protocol can be found in the Supplementary Materials. (d) The 1\% agarose gel showing PCR amplicons that were gel purified vs. non-gel purified. (e) Concentration of dsDNA template after purification and concentration $(n=24)$.

The amplicons with the size that corresponded to the insert with homology arms' size were excised from the gel (Figure 3d) and gel purified. The products were eluted in total of $60 \mu \mathrm{L}$ of nuclease-free water. An additional purification step was used to eliminate any toxic leftovers from the gel as well as concentrate dsDNA. For this step we used Agencourt AMPure's SPRI paramagnetic beads (Beckman Coulter, Pasadena, CA, USA). The final products were eluted in 10-15 $\mu \mathrm{L}$ of nuclease-free water and used in electroporation experiments. This procedure routinely yielded dsDNA at a concentration of $0.9-1.5 \mu \mathrm{g} / \mu \mathrm{L}$ (Figure 3e). At this point, concentrated donor dsDNA was used for the nucleofection (Figure 3b) into activated T cells (Figure 3c), as described in Material and Methods section and the protocol (see Supplementary Materials). 


\subsection{Optimizing Transgene Knock-In in Primary Human T Cells}

After we generated donor DNA, we next proceeded with steps to optimize the knock-in efficiency of the IL-15.E2A.mClover3 transgene in primary human T cells. We tested the following parameters: Template dsDNA concentration, cell numbers, homology arm length, and recovery time.

\subsubsection{DNA Amount}

Electroporation of large amounts of plasmid or linear DNA into cells can be toxic, resulting in poor cell viability and high rates of cell death $[19,22,23]$. To minimize toxicity while maintaining optimal knock-in efficiencies, we first evaluated the effect of different template DNA quantities. We used $1 \mu \mathrm{g}$, $2 \mu \mathrm{g}$, or $3 \mu \mathrm{g}$ of IL-15.E2A.mClover3 DNA in $3 \mu \mathrm{L}$ of nuclease-free water for electroporation together with Cas9: Single-guide (sg) RNA ribonucleoproteins (Cas9 RNPs). Electroporation was performed as shown in Figure $3 \mathrm{~b}$ and described in the Material and Method section. Briefly, activated T cells were resuspended in P3 electroporation buffer and electroporated with $4 \mu \mathrm{L}$ of Cas9 RNPs together with $3 \mu \mathrm{L}$ of donor DNA for HDR. Transgene integration was evaluated 4-6 days later by flow cytometry analysis to determine the percentage of alpha, beta T-cell receptor (TCR $\alpha \beta)$-negative and GFP-positive $\mathrm{T}$ cells (referred to as GFP+ cells thereafter, Figure S1a). Electroporation of T cells with TRAC-specific guide RNA with Cas9 RNPs routinely produced around 91\% efficient knock-out of the TRAC gene as shown in Figure S1b-d. As shown in Figure $4 \mathrm{a}, 1 \mu \mathrm{g}, 2 \mu \mathrm{g}$, or $3 \mu \mathrm{g}$ of template DNA resulted in an average of $21.5 \%, 32.1 \%$, or $29.7 \%$ knock-in efficiency as judged by GFP+ cells (Figure 4a, left panel) with a cell viability of $15.5 \%, 22.4 \%$, or $3.6 \%$, respectively (Figure $4 a$, right panel). Based on this result, we used $2 \mu \mathrm{g}$ of donor DNA in subsequent experiments.

(a)
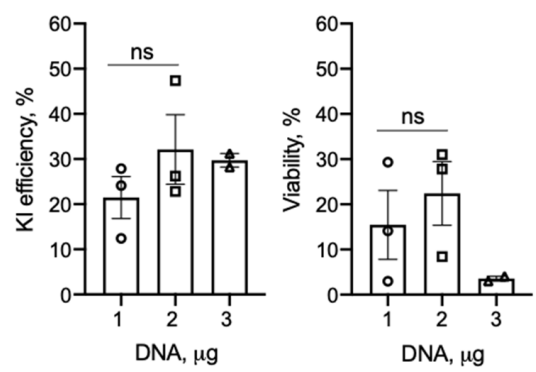

(c)
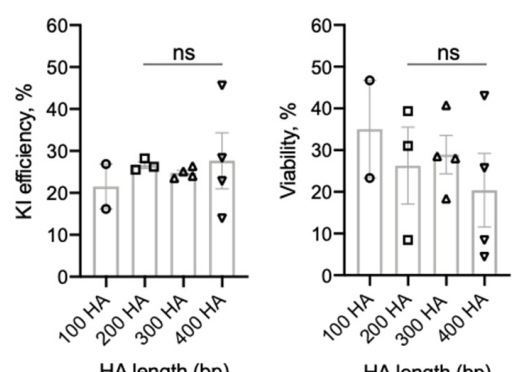
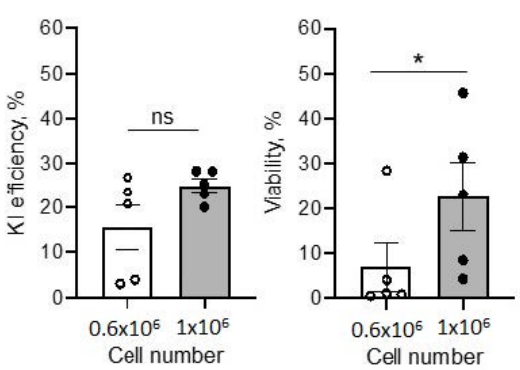

(d)

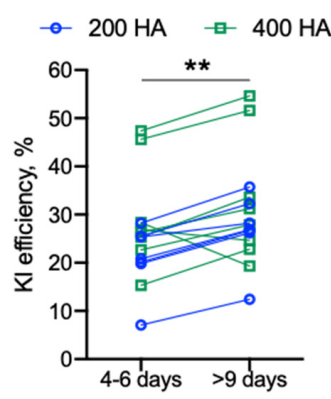

Figure 4. Optimization steps to increase transgene knock-in (KI) efficiency in T cells: (a) Three different dsDNA template concentrations were evaluated for the best knock-in efficiency and cell viability $(n=3$ for 1 and $2 \mu \mathrm{g}, n=2$ for $3 \mu \mathrm{g}$; two-tailed $t$-test; ns-not significant). (b) Two different numbers of $\mathrm{T}$ cells were tested for electroporation $\left(n=5\right.$, two-tailed paired $t$-test, $\left.{ }^{*} p=0.041\right)$. (c) Four different lengths of homology arms flanking the transgene of interest were evaluated $(n=2-4$, one-way ANOVA, ns-not significant). (d) Knock-in efficiency was tested at early (4-6 days) and late ( $>9$ days) time points post-electroporation $\left(n=15\right.$, two-tailed paired $t$-test; $\left.{ }^{* *} p=0.0015\right)$. 


\subsubsection{T-Cell Number}

Next, we tested whether increasing the number of $\mathrm{T}$ cells per reaction improves T-cell recovery post-electroporation and enhances gene editing efficiency. As shown in Figure $4 \mathrm{~b}$, increasing the number of T cells from $0.6 \times 10^{6}$ to $1.0 \times 10^{6}$ per electroporation reaction resulted in a higher KI efficiency $(15.6 \%$ to $25.0 \%$, Figure $4 \mathrm{~b})$; however, it did not reach significance. Importantly, increasing T-cell numbers per reaction significantly $(p=0.04)$ enhanced T-cell survival from $7.0 \%$ to $22.6 \%$, respectively (Figure $4 \mathrm{~b}$ ). As a side note, we also found that combining two electroporation reaction vessels into one recovery well (48-well plate) further improved T-cell viability.

\subsubsection{Homology Arms and Recovery Time}

We next sought to evaluate if longer HA length results in improved knock-in efficiency. To test this, we used our IL-15.E2A.mClover3 transgene flanked by 100, 200, 300, and 400 bp HAs. As shown in Figure 4c, we obtained an average of 21.5, 26.6, 24.8, or 27.7\% GFP+ cells, respectively, with no statistically significant difference between groups. In addition, different HA lengths did not affect T-cell viability (Figure 4c, right panel). Taken together, these results indicate that knock-in efficiency of a large transgene is not dependent on HA length tested.

Finally, we evaluated knock-in efficiency at a later time point ( $>9$ days) post-electroporation, which allows $\mathrm{T}$ cells to rest, recover, and expand. We found that testing for knock-in efficiency at later time points increased the percentage of GFP+ cells in comparison to early (4-6 days) time points (Figure $4 \mathrm{~d}$ ).

\subsection{IL-15 Transgene Is Functional}

To ensure the robustness of our optimized protocol, three different experimenters performed the knock-in assays, routinely achieving an average of 38.2\% KI efficiency (range 26.7-54.6\%, Figure 5a). However, up to this point, we evaluated gene editing efficiency based on GFP+ cells. Since IL-15 is also a component of the transgene, we next tested IL-15 secretion from gene-edited T cells via ELISA. Briefly, $8-10$ days post-electroporation, $5 \times 10^{5}$ cells were washed with phosphate-buffered saline (PBS), resuspended in $275 \mu \mathrm{L}$ of media, and plated in 96-well, V-shaped plates. After $24 \mathrm{~h}, 250 \mu \mathrm{L}$ of media was collected for IL-15 ELISA. As shown in Figure 5b, gene-edited T cells secreted approximately 2 times as much IL-15 compared to control cells (electroporated without DNA, -DNA).
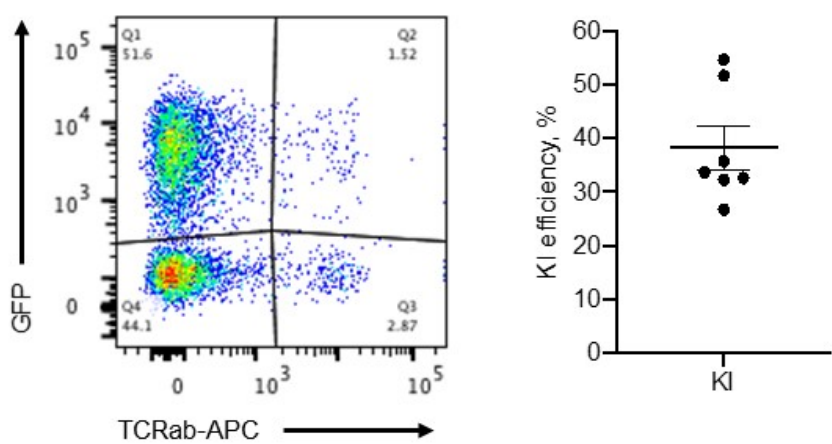

Figure 5. Cont. 
(b)

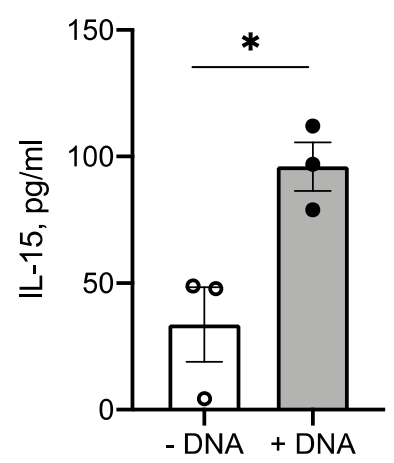

Figure 5. Validating expression of transgenes in gene-edited T cells: (a) Representative flow graph of GFP expression in gene-edited T cells 12 days post-electroporation; right panel, overall knock-in efficiency of the transgene after knock-in optimization as determined by flow cytometry of GFP+/TCR $\alpha \beta$ - cells $(n=7)$. (b) IL-15 production from gene-edited T cells was detected by ELISA 8-10 days post-electroporation $\left(n=3\right.$, two-tailed t-test, $\left.{ }^{*} p=0.024\right)$.

2.6. Application: CAR, BiTE Integration into TRAC Locus and IL-15.E2A.mClover3 Knock-In into IL-13 Locus

Next, we tested if we can knock-in a gene encoding a CAR or bispecific T-cell engager (BiTE) into the TRAC locus using our established protocols. As shown in Figure S2a, we were able to knock-in genes encoding a CAR or BiTE into the TRAC locus with $20 \%$ efficiency.

To test this protocol further, we asked if we could knock-in our IL-15.E2A.mClover3 transgene into a different gene locus. For that purpose, we picked the $I L-13$ locus. We reasoned that, by knocking-in IL-15 into the IL-13 locus, we can also create an inducible system as IL-13 is highly secreted upon T-cell activation (Figure S2b). We used the same IL-15.E2A.mClover3 transgene flanked by homology arms for the $I L-13$ gene locus and performed CRISPR-Cas9-mediated knock-in experiment using our established guidelines. As shown in Figure 6a, we were able to achieve an average of 3\% knock-in as judged by flow cytometry of GFP+ cells. Since IL-13 is activation dependent, we next tested if expression of the transgene is affected by T-cell activation. For that, we activated our gene-edited T cells with ImmunoCult" ${ }^{\mathrm{TM}}$ (Human CD3/CD28/CD2 T Cell Activator, StemCell Technologies, Vancouver, Canada) and quantified GFP+ cells by flow cytometry $24 \mathrm{~h}$ later. Indeed, we observed an average of about 3-fold improvement in knock-in efficiency as judged by GFP+ cells in activated samples when compared to non-activated T cells (Figure $6 \mathrm{~b}$ ). In addition, IL-13 gene knock-out with or without donor DNA led to a significant 2.2-fold decrease in IL-13 secretion ( $p=0.036$ control (ctrl) vs. -DNA and $p=0.007$ ctrl vs. + DNA), indicating successful IL-13 gene disruption (Figure 6c). Finally, we tested IL-15 secretion in IL-13-edited and -activated T cells. As shown in Figure $6 \mathrm{~d}$, we observed a significant increase (average of 2.3-fold) in IL-15 secretion from gene-edited T cells when compared to control T cells. Thus, we can not only knock-in genes of interest into the regions of choice, but also create an inducible system using our optimized protocol. 
(a)
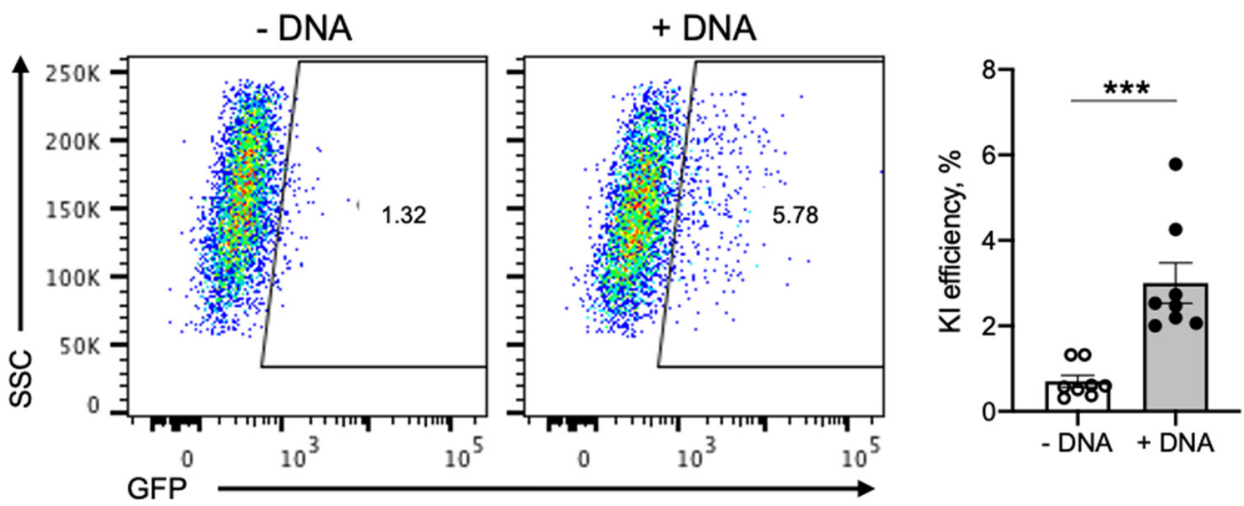

(b)

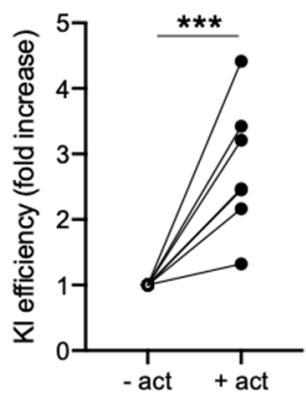

(c)

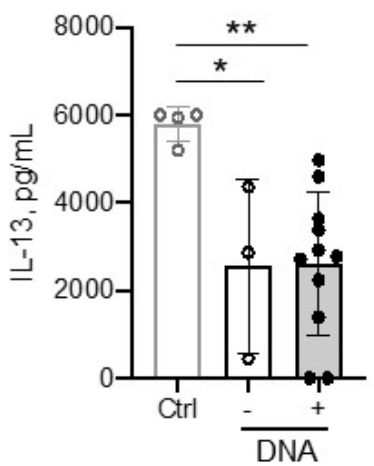

(d)

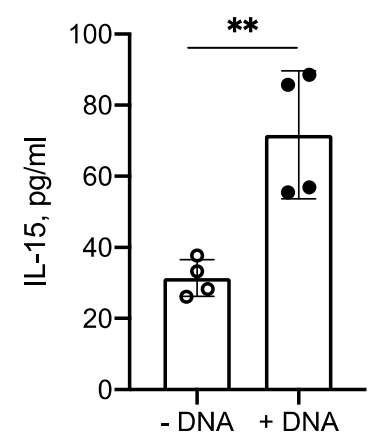

Figure 6. IL-15.E2A.mClover3 knock-in into IL-13 locus: (a) Transgene expression was evaluated by flow cytometry of GFP+ T cells 10 days post-electroporation; left panel, representative flow plots (samples electroporated without template DNA (-DNA) served as controls); right panel, summary graph of left panel $\left(n=8\right.$, two-tailed $t$-test; $\left.{ }^{* * *} p=0.0004\right)$. (b) Fold increase of knock-in efficiency without (-) or with (+) T-cell activation ( $n=6$, two-tailed $t$-test, $\left.{ }^{* * *} p=0.0005\right)$ (c) Knock-out of IL-13 was confirmed by IL-13 secretion; Ctrl, control knock-out; -DNA, knock-in without DNA template; +DNA, knock-in with DNA template $\left(n=3-11\right.$, one-way ANOVA, $\left.{ }^{*} p=0.036^{* *} p=0.007\right)$. (d) IL-15 expression from IL-13-edited (10 days post-electroporation) T cells $24 \mathrm{~h}$ post-T-cell activation $(n=4$, two-tailed $t$-test, $\left.{ }^{* *} p=0.005\right)$.

\section{Discussion}

Here, we demonstrated that primary human T cells can be engineered to express IL-15 and GFP when integrated into the TRAC locus using CRISPR-Cas9 gene editing and non-viral donor DNA as template. In addition, we also showed that we can create an inducible system by inserting IL-15 under the IL-13 promoter to control IL-15 secretion in a T-cell activation-dependent manner.

The ability to generate $\mathrm{T}$ cells expressing a gene-of-interest from a specific locus and/or under a specific promoter opens new avenues for T-cell-based immunotherapies, especially for CAR T-cell-based therapies. Currently, CAR T-cell products are generated mainly by viral transduction, which poses manufacturing challenges as well as safety concerns due to random integration and potential insertional mutagenesis. Here, we provided guidelines on generating template DNA for CRISPR-Cas9-mediated knock-in and performed electroporation to deliver a transgene to the $\mathrm{T}$ cells. We believe that this protocol can serve as a primer for designing and performing knock-in experiments. The level of knock-in efficiency achieved here is sufficient for producing a clinically relevant CAR T-cell product. However, for clinical translation and production, the next step would be to scale up the process to edit $1-3 \times 10^{8} \mathrm{~T}$ cells for future clinical manufacturing. In addition, it is important to mention that in vitro 
and in vivo studies characterizing and evaluating the long-term safety of CRIPSR-Cas9-mediated knock-in are warranted.

As indicated by our data, knock-in efficiency can widely vary and is dependent on gene-of-choice as well as targeted integration site. As a next step of improving editing efficiencies, several adjustments can be considered. For example, Nguyen et al. recently described two improvements to increase knock-in efficiency [24]. They recommend incorporating truncated Cas 9 target sequences at the ends of homology arms. In addition, they also suggested using of polyglutamic acid (PGA), which can stabilize Cas9 RNPs, resulting in an increased editing efficiency while sustaining T-cell viability. However, in our hands, use of PGA improved knock-in efficiency only by $2 \%$. Another modification to consider is the use of single-stranded (ss) DNA instead of dsDNA. The ssDNA triggers a different repair pathway and leads to improved knock-in efficiency and potentially less non-specific integration $[25,26]$. There are multiple commercially available kits for fast and efficient ssDNA generation and several studies described methods on efficient knock-in generation using ssDNA [19,27].

As we demonstrated successful synthetic gene integration into the TRAC locus, we then wanted to apply our established guidelines to integrate $I L-15$ into $I L-13$ locus to potentially design an inducible system. Our initial detection of GFP+ cells was very low, which was expected, given the fact that IL-13 is only expressed/secreted upon T-cell activation. When we activated gene-edited T cells, we observed an increased percentage of GFP+ cells and increased secretion of IL-15, as assessed by ELISA. However, GFP+ cell number and IL-15 secretion were still relatively low. This, in part, can be explained by incomplete knock-out of $I L-13$. Another possible explanation might be that the $I L-13$ promoter might not be strong enough to drive IL-15 expression. To address this, adding a promoter in the DNA template design might solve this issue. However, such approach might render gene expression constitutive; thus, further engineering improvements will have to be considered. Another reason for low efficiency IL-13 locus editing might be the stability of the locus, which in part is mediated by chromatin accessibility/structure. Recently, Sadelain's group reported preliminary data on targeting genomic safe harbors (GSH) [28] and their impact on knock-in efficiency and CAR expression over time. Their findings thus far indicate that not every region targeted for site-specific integration can (1) express exogenous genes and (2) sustain expression over time [29]. This might be the case for the IL-13 locus. To address this, incorporation of insulators or an enhancer might need to be considered when designing donor template.

Although there are already well-established inducible systems regulating transgene expression, such as Syn-Notch and TetON [30-32], one hurdle currently limiting their application is the potential for immunogenicity. By knocking in a transgene into a locus that is expressed only under certain conditions (e.g., IL-13 is only expressed upon T0cell activation), it is possible to use knock-in technology as a non-immunogenic inducible transgene expression system.

We tested multiple variables that can influence T-cell editing efficiency, such as homology arm length, DNA concentration, cell numbers, and time of recovery post-electroporation. We demonstrated that cell number and time of T-cell recovery are important factors for successful large gene integration. Interestingly, our data indicate that there is no difference in knock-in efficiency when using different length of homology arms. This might be due to the size of homology arms we tested. We might observe some differences in editing efficiencies when using longer than $400 \mathrm{bp}$ HAs, such as $800 \mathrm{bp}$ or $1200 \mathrm{bp}$. While this might be beneficial for improving editing efficiencies, having longer HAs will affect DNA concentration, which then will lead to a lower T-cell viability. However, all of these need further experimental testing.

In summary, we developed a reliable protocol to insert genes into the genome of human $\mathrm{T}$ cells using CRISPR-Cas9 gene editing and non-viral donor DNA as template. We believe that it will be useful for investigators as a reference as they embark on using this technology in their laboratories. In addition, we showed that this protocol can be applied for the creation of an inducible expression system. 


\section{Materials and Methods}

\subsection{Generation of DsDNA Donor Template}

\subsubsection{Construct Design}

All constructs for the gene insertion were designed using SnapGene ${ }^{\mathrm{TM}}$ and then synthesized by GeneArt/Life Technologies Corporation (ThermoFisher, Waltham, MA, USA), and subcloned into pMA vector as a final product. A detailed description of IL-15.E2A.mClover3 construct is provided in the Results section. For the CAR knock-in experiment described in the Section 2.6 (Figure S1a), we used

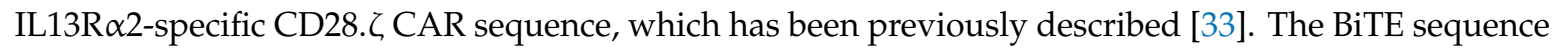
was as follow: TEM8-specific single-chain variable fragment (scFv) L2 [34,35], a short serine-glycine linker, and CD3-specific scFv [36] followed by 2A sequence and Q8 tag for detection. Both constructs contained HAs for TRAC locus.

\subsubsection{PCR Amplification}

Plasmids were transformed and amplified in DH5 $\alpha$ bacterial cells and grown overnight. DNA was extracted by Nucleobond Endotoxin-free Maxiprep (Takara Bio, Mountain View, CA, USA). Primers were designed using SnapGene ${ }^{\mathrm{TM}}$ for different homology arm lengths $(100,200,300$, and $400 \mathrm{bp})$ for insertion in the TRAC locus (Table S1). Rules used for primer design were: $\pm 50 \%$ CG content, less than $22 \mathrm{bp}$ in length, and melting temperatures below $60{ }^{\circ} \mathrm{C}$. The dsDNA was generated by PCR amplification using CloneAmp HiFi Taq polymerase (Takara Bio, Mountain View, CA, USA), forward and reverse primer $(0.5 \mu \mathrm{M})$, plasmid DNA (15-20 ng), and nuclease-free water, in a final volume of $50 \mu \mathrm{L}$. Reactions were run on the ProFlex ${ }^{\mathrm{TM}}$ PCR System (ThermoFisher, Waltham, MA, USA) according to the following program: Initial denaturation at $98^{\circ} \mathrm{C}$ for $30 \mathrm{sec}, 20 \mathrm{cycles}$ of each 3 steps (denaturation at $98{ }^{\circ} \mathrm{C}$ for $10 \mathrm{sec}$, annealing at $+3{ }^{\circ} \mathrm{C}$ of lower melting temperature of primer for $15 \mathrm{sec}$, and extension at $72{ }^{\circ} \mathrm{C}$ for variable time based on PCR product size $-5 \mathrm{sec} / \mathrm{kb}$ ), final extension at $72{ }^{\circ} \mathrm{C}$ for $3 \mathrm{~min}$. Two PCR reactions combined were run on $1 \%$ agarose gel for band size confirmation. The BenchTop $1 \mathrm{~kb}$ DNA Ladder (Promega, Madison, WI, USA) was used in all experiments. To generate highly concentrated dsDNA, 8 PCR reactions were run in total and 2 of these reactions were combined in one gel slot.

\subsubsection{DNA Purification and Concentration}

The amplicons with the size-corresponding transgene DNA size were excised from the gel and purified using the NucleoSpin ${ }^{\circledR}$ Gel and PCR clean up kit (Takara Bio, Mountain View, CA, USA). The products were eluted in $60 \mu \mathrm{L}$ of nuclease-free water and used for further concentration. An additional purification step was used to eliminate any toxic leftovers from the gel. For this step, we used Agencourt AMPure's SPRI paramagnetic bead technology (Beckman Coulter, Pasadena, CA, USA). The final products were eluted in 10-15 $\mu \mathrm{L}$ of nuclease-free water (to get a final concentration of $\sim 1-1.5 \mu \mathrm{g} / \mu \mathrm{L}$ ) and used in electroporation experiments.

\subsection{Generation of Knock-In T Cells}

\subsubsection{Primary Human T-Cell Culture}

Human peripheral blood mononuclear cells (PBMCs) were obtained from whole blood of healthy donors under the Institutional Review Board (IRB)-approved protocols at St. Jude Children's Research Hospital. To generate gene-edited T cells, we isolated PBMCs by Lymphoprep (Abbott Laboratories, Chicago, IL, USA) gradient centrifugation. CD4+/CD8+ T cells were then enriched from the PBMCs using human anti-CD4- and anti-CD8-specific MicroBeads kit (Miltenyi Biotec, Bergisch Gladbach, Germany), according to the manufacturer's protocol. Enriched T cells were plated in a 24-well, non-tissue culture-treated plate at $0.5 \times 10^{6}$ cells/mL in $2 \mathrm{~mL}$ T-cell media (PMI (GE Healthcare Life 
Sciences, Marlborough, MA, USA) containing 10\% fetal bovine serum (FBS) (GE Healthcare Life Sciences, Marlborough, MA, USA), and 1\% GlutaMAX-I (Invitrogen, Carlsbad, CA, USA). The next day, selected T cells were stimulated with $25 \mu \mathrm{L}$ Human T-Activator CD3- and CD28-specific Dynabeads (ThermoFisher, Waltham, MA, USA) and grown in the T-cell media supplemented with recombinant human IL-7 and IL-15 cytokines (IL-7: 10 ng/mL, IL-15: 5 ng/mL, PeproTech Cranbury, NJ, USA).

\subsubsection{Primary Human T-Cell Electroporation}

Two days after T-cell activation, cells were electroporated to enable site-specific knock-in using Cas9 RNPs. All electroporation experiments were performed on the $4 \mathrm{D}-$ Nucleofector ${ }^{\mathrm{TM}}$ System X Unit (Lonza, Basel, Switzerland) using the EH-115 program. RNPs were pre-complexed at a sgRNA:Cas9 ratio of 4.5:1, prepared by adding $3 \mu \mathrm{L}$ of $60 \mu \mathrm{M}$ sgRNA (Synthego Menlo Park, CA, USA) to $1 \mu \mathrm{L}$ of $40 \mu \mathrm{M}$ Cas 9 (QB3 Macrolab, University of California, Berkeley, CA, USA), and incubated for $10 \mathrm{~min}$ at room temperature (RT). Complexed RNPs were used right away or frozen for later use. Sequences for all sgRNAs can be found in Table S2. T cells $\left(0.6 \times 10^{6}\right.$ or $\left.1.0 \times 10^{6}\right)$ were re-suspended in $17 \mu \mathrm{L}$ P3 buffer including supplement 1 (Lonza). Subsequently, $4 \mu \mathrm{L}$ of RNP complex was added together with the dsDNA template donor ( $2 \mu \mathrm{g} / 3 \mu \mathrm{L}$ unless stated otherwise) and incubated for $10 \mathrm{~min}$ at room temp. The RNP and dsDNA mix were added to the cell mixture and $23 \mu \mathrm{L}$ was added to the transfection vessel and electroporated. After electroporation, $80 \mu \mathrm{L}$ of recovery media (RPMI (GE Healthcare Life Sciences, Marlborough, MA, USA) including 20\% FBS (GE Healthcare Life Sciences, Marlborough, MA, USA), $1 \%$ GlutaMAX-I (Invitrogen, Carlsbad, CA, USA), IL-7 at $10 \mathrm{ng} / \mathrm{mL}$, and IL- 15 at $5 \mathrm{ng} / \mathrm{mL}$ ) was added to the electroporation vessel. The cells were rested for $30 \mathrm{~min}$ at $37^{\circ} \mathrm{C}$ and $5 \% \mathrm{CO}_{2}$ before being transferred into a 48 -well, tissue culture plate with $650 \mu \mathrm{L}$ of recovery media. Two to three days after electroporation, the FBS concentration was reduced to $10 \%$ in the T-cell culture media with cytokines. T cells were split every 3-4 days and fresh IL-7 and IL-15 cytokines were added.

\subsection{Flow Cytometry}

Cells were examined by flow cytometry 4 to 6 or $>9$ days after electroporation to determine the knock-out and knock-in efficiencies of the desired gene constructs. All flow cytometry experiments were performed on the FACSCanto ${ }^{\mathrm{TM}}$ instruments (BD Bioscience, San Jose, CA, USA). FACSDiva (BD Biosciences, San Jose, CA, USA) and FlowJo v.10 (FlowJo, Ashland, OR, USA) were used for analyzing the acquired immunofluorescence data. For surface staining, samples were washed with and stained in PBS (Lonza) with 1\% FBS (GE Healthcare Life Sciences, Marlborough, MA, USA). For all experiments, matched isotypes or known negatives (e.g., non-edited or knock-out only T cells) served as gating controls.

Live cells' populations were evaluated based on SSC-A over FSC-A gating [37] or using LIVE/DEAD Fixable Aqua Dead Cell Stain Kit (Invitrogen, Carlsbad, CA, USA) as a viability dye. TRAC expression was determined by using a mouse anti-human TCR $\alpha \beta$-APC or TCR $\alpha \beta$-PE antibody (BD Biosciences, San Jose, CA, USA). The mClover3, which is protein with a higher fluorescence signal of a jellyfish GFP, positive cells (referred to as GFP+ cell in the text) were detected in the GFP channel [21]. Detection of IL13R $\alpha 2-C A R$ was achieved with recombinant human IL13R $\alpha 2$ protein conjugated to PE (Creative BioMart, Shirley, NY, USA). Expression of Q8 was detected using anti-human CD34 (Qbend 10) APC antibody (R\&D Systems, Minneapolis, MN, USA).

\subsection{Targeted Deep Sequencing}

The hTRAC-specific amplicons were generated using gene-specific primers with partial Illumina adapter overhangs (hTRAC.F - 5'-AGTGTAATACCTTGCAGCACCAGAGC-3' and hTRAC.R 5'-TTGCTCCAGGCCACAGCACTGTTGC-3' ${ }^{\prime}$, overhangs not shown) as previously described [38]. Briefly, hTRAC-specific amplicons were generated, indexed, and pooled with other targeted amplicons for other loci. Additionally, 10\% PhiX Sequencing Control V3 (Illumina, San Diego, CA, USA) was added to the pooled amplicon library prior to running the sample on an Illumina Miseq sequencer to 
generate paired $2 \times 250$ reads. Samples were demultiplexed using the index sequences, fastq files were generated, and Next-Generation Sequencing (NGS) analysis was performed using CRIS.py [39].

\subsection{Analysis of IL-15 and IL-13 Production}

IL-15 production was measured using a Human IL-15 Quantikine ELISA Kit (R\&D Systems, Minneapolis, MN, USA) according to the manufacturer's protocol. IL-13 production was analyzed using Human IL-13 Quantikine ELISA Kit (R\&D Systems, Minneapolis, MN, USA). For measuring IL-15 secretion upon T-cell activation, $5.0 \times 10^{5} \mathrm{~T}$ cells were washed with PBS, resuspended in $275 \mu \mathrm{L}$ of T-cell media (RPMI, 10\% FBS, and 1\% GlutaMAX) without cytokines and plated in 96-well, V-shaped plates. Cells were then activated with Immunocult ${ }^{\mathrm{TM}}$ Human CD3/CD28/CD2 T Cell Activator (StemCell Technologies, Vancouver, Canada) following the manufacturer's protocol and incubated at $37^{\circ} \mathrm{C}$. After $24 \mathrm{~h}, 250 \mu \mathrm{L}$ of media was collected for IL-15 ELISA from the wells and stored at $-80^{\circ} \mathrm{C}$.

\subsection{Statistical Analysis}

All statistical analyses were performed in GraphPad PRISM 8 (GraphPad Software, San Diego, CA, USA). All experiments were performed at least in duplicates. For comparison between two groups, two-tailed $t$-test was used. For comparisons of three or more groups, values were log transformed as needed and analyzed by ANOVA. $p$ values $<0.05$ were considered statistically significant.

\section{Conclusions}

CRISPR-Cas9 knock-in approaches allow for efficient and fast site-specific gene integration in primary human $\mathrm{T}$ cells when donor DNA is provided in non-viral form. This allows for efficient generation of gene-edited T cells expressing multiple therapeutically relevant genes. Here we provided guidelines to streamline donor DNA design and maximize editing efficiency for CRISPR-Cas9 gene editing (Table 1).

Table 1. Electroporation checklist for large gene knock-in in human T cells.

\begin{tabular}{cc}
\hline sgRNA & $3 \mu \mathrm{L}[60 \mu \mathrm{M}]$ \\
\hline Cas9 & $1 \mu \mathrm{L}[40 \mu \mathrm{M}]$ \\
\hline sgRNA:Cas9 (molar ratio) & $4.5: 1$ \\
\hline RNP incubation & $10 \mathrm{~min}, \mathrm{RT}$ \\
\hline RNP volume & $4 \mu \mathrm{L}$ \\
\hline Cells & $1 \times 10^{6} / 17 \mu \mathrm{L}$ \\
\hline Electroporation solution & $\mathrm{P} 3+\mathrm{S} 1$ supplement $(\mathrm{Lonza})$ \\
\hline Template & $2 \mu \mathrm{g}$ dsDNA in $3 \mu \mathrm{L}$ \\
\hline Homology arm length & $23 \mu \mathrm{L}$ \\
\hline Vol for electroporation & Strip/EH-115 \\
\hline Format/program Neon & 30 min at $37^{\circ} \mathrm{C}($ in $80 \mu \mathrm{L} \mathrm{of} \mathrm{RPMI+20 \% FBS+IL7/15)}$ \\
\hline Incubation after electroporation & 48 -well plate $(650 \mu \mathrm{L}$ of RPMI+20\%FBS+IL7/15) \\
\hline Transfer & 2 \\
\hline Reactions per well after electroporation & \\
\hline
\end{tabular}

Supplementary Materials: The following are available online at http://www.mdpi.com/2072-6694/12/6/1704/s1, Figure S1. IL-15.E2A.mClover3 transgene integration into TRAC locus: (a) Gating strategy for detection of transgene expression in T cells by flow cytometry. (b) Knock-out efficiency of TCR in T cells without (-) or with (+) dsDNA template as measured by flow cytometry $(n=5-12)$. (c) Editing of TRAC locus with gRNA (Eyquem et al., 2017) as determined by targeted NGS, $n=3$. (d) Summary of the most frequent indels by deep sequencing 
after editing of TCR locus in T cells (sample representative from $n=3$ ). The asterisk indicates an unedited allele. Figure S2. Applications of site-specific transgene integration using CRISPR-Cas9: (a) Successful knock-in of a CAR and a BiTE into the TRAC locus was confirmed by flow cytometry. For details on CAR and BiTE sequences, see Section 4.1.1. (b) IL-13 production is increased upon T-cell activation (-act, non-activated; +act, activated) $(n=$ 3). Knock-In Protocol: Table S1. Primer sequence for dsDNA template. Table S2. SgRNA sequences for TRC locus. Complete and detailed knock-in protocol can be found in Supplementary Materials.

Author Contributions: Conceptualization, G.K. and Z.O.; methodology, Z.O. and G.K.; formal analysis, Z.O., J.C., and G.K.; investigation, Z.O., J.C., and N.P.; data curation, Z.O., J.C., and G.K.; writing-original draft preparation, Z.O., J.C., and G.K.; writing-review and editing, Z.O., J.C., N.P., S.Z., G.K., and Z.O.; supervision, G.K.; funding acquisition, G.K. All authors have read and agreed to the published version of the manuscript.

Funding: This work was supported by the Assisi Foundation of Memphis and the American Lebanese Syrian Associated Charities (ALSAC).

Acknowledgments: We would like to thank Stephen Gottschalk for constructive discussions and review of our manuscript. We would like to thank all members of the Dept. of Bone Marrow Transplantation and Cellular Therapy at St. Jude Children's Research Hospital for their critical scientific inputs and discussions. We thank the Center for Advanced Genome Engineering (CAGE), which is supported by the National Cancer Institute (NCI, USA) P30 CA021765 grant for the targeted deep sequencing library preparation and analysis. Illustrations in Figure 3 were created with BioRender.com.

Conflicts of Interest: G.K. has patent applications in the field of immunotherapy.

\section{References}

1. Maldini, C.R.; Ellis, G.I.; Riley, J.L. CAR T cells for infection, autoimmunity and allotransplantation. Nat. Rev. Immunol. 2018, 18, 605-616. [CrossRef] [PubMed]

2. Sadelain, M.; Riviere, I.; Riddell, S. Therapeutic T cell engineering. Nature 2017, 545, 423-431. [CrossRef] [PubMed]

3. Maude, S.L.; Frey, N.; Shaw, P.A.; Aplenc, R.; Barrett, D.M.; Bunin, N.J.; Chew, A.; Gonzalez, V.E.; Zheng, Z.; Lacey, S.F.; et al. Chimeric antigen receptor T cells for sustained remissions in leukemia. N. Engl. J. Med. 2014, 371, 1507-1517. [CrossRef] [PubMed]

4. Davila, M.L.; Riviere, I.; Wang, X.; Bartido, S.; Park, J.; Curran, K.; Chung, S.S.; Stefanski, J.; Borquez-Ojeda, O.; Olszewska, M.; et al. Efficacy and toxicity management of 19-28z CAR T cell therapy in B cell acute lymphoblastic leukemia. Sci. Transl. Med. 2014, 6, 224ra25. [CrossRef] [PubMed]

5. Lee, D.W.; Kochenderfer, J.N.; Stetler-Stevenson, M.; Cui, Y.K.; Delbrook, C.; Feldman, S.A.; Fry, T.J.; Orentas, R.; Sabatino, M.; Shah, N.N.; et al. T cells expressing CD19 chimeric antigen receptors for acute lymphoblastic leukaemia in children and young adults: A phase 1 dose-escalation trial. Lancet 2015, 385, 517-528. [CrossRef]

6. Gardner, R.A.; Finney, O.; Annesley, C.; Brakke, H.; Summers, C.; Leger, K.; Bleakley, M.; Brown, C.; Mgebroff, S.; Kelly-Spratt, K.S.; et al. Intent-to-treat leukemia remission by CD19 CAR T cells of defined formulation and dose in children and young adults. Blood 2017, 129, 3322-3331. [CrossRef]

7. Hacein-Bey-Abina, S.; Von Kalle, C.; Schmidt, M.; McCormack, M.P.; Wulffraat, N.; Leboulch, P.; Lim, A.; Osborne, C.S.; Pawliuk, R.; Morillon, E.; et al. LMO2-associated clonal T cell proliferation in two patients after gene therapy for SCID-X1. Science 2003, 302, 415-419. [CrossRef]

8. Nam, C.H.; Rabbitts, T.H. The role of LMO2 in development and in T cell leukemia after chromosomal translocation or retroviral insertion. Mol. Ther. 2006, 13, 15-25. [CrossRef]

9. van der Loo, J.C.; Wright, J.F. Progress and challenges in viral vector manufacturing. Hum. Mol. Genet. 2016, 25, R42-R52. [CrossRef]

10. Merten, O.W.; Charrier, S.; Laroudie, N.; Fauchille, S.; Dugué, C.; Jenny, C.; Audit, M.; Zanta-Boussif, M.A.; Chautard, H.; Radrizzani, M.; et al. Large-scale manufacture and characterization of a lentiviral vector produced for clinical ex vivo gene therapy application. Hum. Gene Ther. 2011, 22, 343-356. [CrossRef]

11. Wright, J.F. Manufacturing and characterizing AAV-based vectors for use in clinical studies. Gene Ther. 2008, 15, 840-848. [CrossRef]

12. David, R.M.; Doherty, A.T. Viral Vectors: The Road to Reducing Genotoxicity. Toxicol. Sci. 2017, 155, 315-325. [CrossRef] [PubMed]

13. Tyagarajan, S.; Schmitt, D.; Acker, C.; Rutjens, E. Autologous cryopreserved leukapheresis cellular material for chimeric antigen receptor-T cell manufacture. Cytotherapy 2019, 21, 1198-1205. [CrossRef] [PubMed] 
14. Webber, B.R.; Lonetree, C.; Kluesner, M.G.; Johnson, M.J.; Pomeroy, E.J.; Diers, M.D.; Lahr, W.S.; Draper, G.M.; Slipek, N.J.; Smeester, B.A.; et al. Highly efficient multiplex human T cell engineering without double-strand breaks using Cas9 base editors. Nat. Commun. 2019, 10, 5222. [CrossRef]

15. Schumann, K.; Lin, S.; Boyer, E.; Simeonov, D.R.; Subramaniam, M.; Gate, R.E.; Haliburton, G.E.; Ye, C.J.; Bluestone, J.A.; Doudna, J.A.; et al. Generation of knock-in primary human T cells using Cas9 ribonucleoproteins. Proc. Natl. Acad. Sci. USA 2015, 112, 10437-10442. [CrossRef] [PubMed]

16. Xu, X.; Gao, D.; Wang, P.; Chen, J.; Ruan, J.; Xu, J.; Xia, X. Efficient homology-directed gene editing by CRISPR/Cas9 in human stem and primary cells using tube electroporation. Sci. Rep. 2018, 8, 11649. [CrossRef] [PubMed]

17. Hale, M.; Lee, B.; Honaker, Y.; Leung, W.-H.; Grier, A.E.; Jacobs, H.M.; Sommer, K.; Sahni, J.; Jackson, S.W.; Scharenberg, A.M.; et al. Homology-Directed Recombination for Enhanced Engineering of Chimeric Antigen Receptor T Cells. Mol. Ther. Methods Clin. Dev. 2017, 4, 192-203. [CrossRef]

18. Eyquem, J.; Mansilla-Soto, J.; Giavridis, T.; van der Stegen, S.J.C.; Hamieh, M.; Cunanan, K.M.; Odak, A.; Gönen, M.; Sadelain, M. Targeting a CAR to the TRAC locus with CRISPR/Cas9 enhances tumour rejection. Nature 2017, 543, 113-117. [CrossRef]

19. Roth, T.L.; Puig-Saus, C.; Yu, R.; Shifrut, E.; Carnevale, J.; Li, P.J.; Hiatt, J.; Saco, J.; Krystofinski, P.; Li, H.; et al. Reprogramming human $\mathrm{T}$ cell function and specificity with non-viral genome targeting. Nature 2018, 559, 405-409. [CrossRef]

20. MacLeod, D.T.; Antony, J.; Martin, A.J.; Moser, R.J.; Hekele, A.; Wetzel, K.J.; Brown, A.E.; Triggiano, M.A.; Hux, J.A.; Pham, C.D.; et al. Integration of a CD19 CAR into the TCR Alpha Chain Locus Streamlines Production of Allogeneic Gene-Edited CAR T Cells. Mol. Ther. 2017, 25, 949-961. [CrossRef] [PubMed]

21. Bajar, B.T.; Wang, E.S.; Lam, A.J.; Kim, B.B.; Jacobs, C.L.; Howe, E.S.; Davidson, M.W.; Lin, M.Z.; Chu, J. Improving brightness and photostability of green and red fluorescent proteins for live cell imaging and FRET reporting. Sci. Rep. 2016, 6, 20889. [CrossRef] [PubMed]

22. Cornu, T.I.; Mussolino, C.; Cathomen, T. Refining strategies to translate genome editing to the clinic. Nat. Med. 2017, 23, 415-423. [CrossRef] [PubMed]

23. Luecke, S.; Holleufer, A.; Christensen, M.H.; Jønsson, K.L.; Boni, G.A.; Sørensen, L.K.; Johannsen, M.; Jakobsen, M.R.; Hartmann, R.; Paludan, S.R. cGAS is activated by DNA in a length-dependent manner. EMBO Rep. 2017, 18, 1707-1715. [CrossRef] [PubMed]

24. Nguyen, D.N.; Roth, T.L.; Li, P.J.; Chen, P.A.; Apathy, R.; Mamedov, M.R.; Vo, L.T.; Tobin, V.R.; Goodman, D.; Shifrut, E.; et al. Polymer-stabilized Cas9 nanoparticles and modified repair templates increase genome editing efficiency. Nat. Biotechnol. 2020, 38, 44-49. [CrossRef] [PubMed]

25. Kan, Y.; Ruis, B.; Takasugi, T.; Hendrickson, E.A. Mechanisms of precise genome editing using oligonucleotide donors. Genome Res. 2017, 27, 1099-1111. [CrossRef] [PubMed]

26. Liu, M.; Rehman, S.; Tang, X.; Gu, K.; Fan, Q.; Chen, D.; Ma, W. Methodologies for Improving HDR Efficiency. Front. Genet. 2018, 9, 691. [CrossRef]

27. Paix, A.; Folkmann, A.; Goldman, D.H.; Kulaga, H.; Grzelak, M.J.; Rasoloson, D.; Paidemarry, S.; Green, R.; Reed, R.R.; Seydoux, G. Precision genome editing using synthesis-dependent repair of Cas9-induced DNA breaks. Proc. Natl. Acad. Sci. USA 2017, 114, E10745-E10754. [CrossRef]

28. Sadelain, M.; Papapetrou, E.P.; Bushman, F.D. Safe harbours for the integration of new DNA in the human genome. Nat. Rev. Cancer 2011, 12, 51-58. [CrossRef]

29. Odak, A.; Yuan, H.; Mansilla-Soto, J.; Eyquem, J.; Vedantam, P.; Leslie, C.; Sadelain, M. ASGCT Abstract Book. Abstract 941: Novel Genomic Safe Harbors for Effective CAR T Cell Engineering. Mol. Ther. 2019, $27,1$.

30. Morsut, L.; Roybal, K.T.; Xiong, X.; Gordley, R.M.; Coyle, S.M.; Thomson, M.; Lim, W.A. Engineering Customized Cell Sensing and Response Behaviors Using Synthetic Notch Receptors. Cell 2016, 164, 780-791. [CrossRef]

31. Roybal, K.T.; Williams, J.Z.; Morsut, L.; Rupp, L.J.; Kolinko, I.; Choe, J.H.; Walker, W.J.; McNally, K.A.; Lim, W.A. Engineering T Cells with Customized Therapeutic Response Programs Using Synthetic Notch Receptors. Cell 2016, 167, 419-432.e16. [CrossRef] [PubMed]

32. Das, A.T.; Tenenbaum, L.; Berkhout, B. Tet-On Systems for Doxycycline-inducible Gene Expression. Curr. Gene Ther. 2016, 16, 156-167. [CrossRef] [PubMed] 
33. Krenciute, G.; Krebs, S.; Torres, D.; Wu, M.; Liu, H.; Dotti, G.; Li, X.-N.; Lesniak, M.S.; Balyasnikova, I.V.; Gottschalk, S. Characterization and Functional Analysis of scFv-based Chimeric Antigen Receptors to Redirect T Cells to IL13Ralpha2-positive Glioma. Mol. Ther. 2016, 24, 354-363. [CrossRef] [PubMed]

34. Chaudhary, A.; BethHilton, M.; Seaman, S.; Haines, D.C.; Stevenson, S.; Lemotte, P.K.; Tschantz, W.R.; Zhang, X.M.; Saha, S.; Fleming, T.; et al. TEM8/ANTXR1 blockade inhibits pathological angiogenesis and potentiates tumoricidal responses against multiple cancer types. Cancer Cell 2012, 21, 212-226. [CrossRef]

35. Williams, L.; Lundqvist, A.; van Hoef, V.; Zhang, X.; Wennerberg, E.; Lorent, J.; Witt, K.; Sanz, L.M.; Liang, S.; Murray, S.; et al. 31st Annual Meeting and Associated Programs of the Society for Immunotherapy of Cancer (SITC 2016): P51 T cells redirected to TEM8 have antitumor activity but induce 'on target/off cancer toxicity' in preclinical models. In Journal for Immunotherapy of Cancer; Springer: Berlin/Heidelberg, Germany, 2016.

36. Iwahori, K.; Kakarla, S.; Velasquez, M.P.; Yu, F.; Yi, Z.; Gerken, C.; Song, X.-T.; Gottschalk, S. Engager T cells: A new class of antigen-specific T cells that redirect bystander T cells. Mol. Ther. 2015, 23, 171-178. [CrossRef]

37. Bohmer, R.M.; Bandala-Sanchez, E.; Harrison, L.C. Forward light scatter is a simple measure of T-cell activation and proliferation but is not universally suited for doublet discrimination. Cytometry A 2011, 79, 646-652. [CrossRef] [PubMed]

38. Sentmanat, M.F.; Peters, S.T.; Florian, C.P.; Connelly, J.P.; Pruett-Miller, S.M. A Survey of Validation Strategies for CRISPR-Cas9 Editing. Sci. Rep. 2018, 8, 888. [CrossRef] [PubMed]

39. Connelly, J.P.; Pruett-Miller, S.M. CRIS.py: A Versatile and High-throughput Analysis Program for CRISPR-based Genome Editing. Sci. Rep. 2019, 9, 4194. [CrossRef]

(C) 2020 by the authors. Licensee MDPI, Basel, Switzerland. This article is an open access article distributed under the terms and conditions of the Creative Commons Attribution (CC BY) license (http://creativecommons.org/licenses/by/4.0/). 